\title{
APROXIMACIONES PRAGMÁTICAS A LA CREENCIA Y LA VERDAD
}

\author{
Marco Antonio Joven-Romero \\ Universidad de Santo Tomás (Manila) \\ majovenromero@bec.uned.es
}

\section{RESUMEN}

Bastantes filósofos están explorando la relación entre creencia y verdad, siguiendo la popular frase de Williams la creencia aspira a la verdad. Normativistas apoyan una relación constitutiva entre creencia y verdad en términos de la norma de la verdad. Teleologistas lo hacen en términos del objetivo de la verdad y los mecanismos que regulan la verdad. Otros analizan la creencia y la verdad desde el pragmatismo. Aquí analizo estas ideas sobre creencia, verdad y pragmatismo, defendiendo que la creencia puede entenderse mejor considerando una relación constitutiva con la verdad. Las posiciones pragmáticas podrían ser mejores para comprender aceptación.

Palabras Clave: normatividad, teleologismo, pragmatismo, aceptación, wishful thinking.

PRAGMATIC APPROACHES TO BELIEF AND TRUTH

\section{Abstract}

Many philosophers explore the relationship between belief and truth, following Williams popular statement "belief aims at truth." Normativists support a constitutive link between belief and truth in terms of the norm of truth while the teleologists do it in terms of the aim of truth and the mechanisms that regulate truth. Other philosophers analyze belief and truth from pragmatism. Here I analyze these ideas about belief, truth and pragmatism defending that belief can be better understood with a constitutive relationship between belief and truth. The pragmatic positions might be better to understand and manage acceptance. KEYWORDS: normativity, teleologism, pragmatism, acceptance, wishful thinking. 


\section{INTRODUCCIÓN}

En los últimos años, algunos filósofos están analizando las relaciones entre creencia y verdad, encontrando un punto de inicio en la popular frase de Williams ${ }^{1}$ la creencia aspira a la verdad. Las aproximaciones teleológicas establecen que la creencia aspira a la verdad como objetivo: si un estado o actitud proposicional no apunta a la verdad, entonces no es una creencia. El teleologismo se puede interpretar desde los objetivos de los agentes, y, entre ellos, el objetivo de la verdad: la creencia aspira a la verdad porque el agente ha formado su creencia precisamente para ser una creencia verdadera. También se puede interpretar el teleologismo en términos de los mecanismos de formación de la creencia: la creencia aspira a la verdad porque el agente desarrolla y tiene (por evolución o entrenamiento) mecanismos para la formación de creencias verdaderas.

Las aproximaciones normativas establecen que los agentes deben aceptar una norma que juzga a las creencias en términos de la verdad. El estándar de corrección de la creencia es la norma de la verdad. Hay distintas opciones para formular esta norma ${ }^{2}$. Tanto los normativistas como muchos de los teleologistas están de acuerdo en que el vínculo entre la creencia y la verdad es constitutivo, aunque difieren a la hora de tratar la naturaleza de este vínculo. Los normativistas inquieren a los teleologistas por la naturaleza del mecanismo que regula la creencia, en tanto en cuanto los normativistas lo explican a partir de normas intrínsecas a la creencia (por ejemplo: un agente debe creer $p$ si y solo si $p$ es verdad). Pero una norma doxástica fuerte sobre la creencia hace difícil explicar por qué las creencias no siempre reflejan la verdad. Por ello, las normas propuestas se suelen centrar en la naturaleza de la «aspiración» como proceso (la creencia aspira a la verdad), y no en los resultados de las creencias (esto es, hasta qué punto la creencia refleja la realidad, el conocimiento o la verdad). Los teleologistas podrían preguntar a los normativistas qué diferencias hay entre considerar la norma de aspirar a la verdad y directamente la aspiración a la verdad. Para el normativista, la norma se podría explicar en términos de algún tipo de internalismo motivacional y el teleologista podría replicar analizando la aspiración como proceso en términos de evolución y entrenamiento. Si el agente desarrolla una creencia que no aspira a la verdad, los normativistas dirían que el agente ha violado la norma debido a un comportamiento irracional, mientras que los teleologistas dirían que los mecanismos evolutivos y de entretamiento no han funcionado como deberían. Finalmente, las posturas normativistas acaban descansando en la racionalidad del agente, mientras que el teleologismo no necesita apelar a ella.

1 Williams, B. Problems of the Self, Cambridge University Press, Cambridge, 1973.

${ }^{2}$ La más común: «La creencia debe aspirar a la verdad». Otra, que intenta salvar algunos problemas que presenta la anterior, es la de que «la creencia puede aspirar a la verdad» «la creencia no debe aspirar a la mentira», Whiting, D. «Should I Believe the Truth?», Dialectica, 64 (2), 2010, pp. 213-224. Whiting, D. «Nothing but the Truth: On the Norms and Aims of Belief», The Aim of Belief, Oxford University Press, Oxford, 2013b, pp. 184-203. 
Contrariamente a estas opciones, otros filósofos no admiten un estatus privilegiado de la verdad en la creencia, rechazando así una relación constitutiva entre la creencia y la verdad. La verdad no es el único criterio para la creencia, y en los casos más radicales, la verdad no existe en la creencia o es reducible a otras consideraciones o justificaciones. Esto abre la puerta a nuevas opciones como la creencia aspirando a consideraciones pragmáticas o la creencia aspirando a su justificación. Entre estas consideraciones, presto especial atención al análisis pragmático de la relación entre la creencia y la verdad. Otros autores defienden que hay una relación constitutiva entre la creencia y la verdad, pero al mismo tiempo relacionan la verdad con consideraciones pragmáticas: lo que es verdad es práctico.

En este artículo primero discuto el análisis de algunos autores contemporáneos sobre la creencia, la verdad y el pragmatismo. Algunas veces estos análisis están directamente relacionados con el pragmatismo, otras veces involucran ideas que pueden ser consideradas desde un punto de vista pragmático aunque no se mencione explícitamente. Después, tomo y aplico las ideas de algunos filósofos pragmáticos y neopragmáticos sobre la verdad, establecidas durante el último tercio del siglo xx y principios del siglo XxI. Expongo cómo estas ideas pueden contribuir a encarar y afrontar algunos problemas típicos y finalmente defiendo que una relación constitutiva entre creencia y verdad es necesaria para explicar la naturaleza de estos conceptos.

\section{LA CREENCIA Y CONSIDERACIONES PRÁCTICAS}

En esta sección discuto las ideas sobre el pragmatismo y la creencia (y en menor medida, también la verdad) de algunos autores contemporáneos: Papineau ${ }^{3}$, Owens $^{4}$, Engel ${ }^{5}$, Whiting ${ }^{6}, \mathrm{McHugh}^{7}$ y Unwin ${ }^{8}$. Algunos de estos autores defienden posiciones normativas (Owens, Engel, Whiting y McHugh), mientras que otros están más próximos a las posiciones pragmáticas (Papineau, Unwin). En cualquier caso, la

3 Papineau, D. «There Are No Norms of Belief», The Aim of Belief, Oxford University Press, Oxford, 2013, pp. 64-79.

4 Owens, D. «Value and Epistemic Normativity», Teorema: Revista internacional de filosofía, 32 (3), 2013, pp. 35-58.

5 Engel, P. «Belief and the Right Kind of Reason», Teorema: Revista internacional de filosofia, 32(3), 2013a, pp. 19-34. ENGEL, P. «In Defense of Normativism about the Aim of Belief», The Aim of Belief, Oxford University Press, Oxford, 2013b, pp. 43-85.

6 Whiting, D. «Should I Believe the Truth?», Dialectica, 64 (2), 2010, pp. 213-224. Whiting, D. «Truth: the Aim and Norm of Belief», Teorema: Revista internacional de filosofía, 32 (3), 2013a, pp. 121-135. Whiting, D. «Nothing but the Truth: On the Norms and Aims of Belief», The Aim of Belief, Oxford University Press, Oxford, 2013b, pp.184-203. Whiting, D. «Reasons for Belief, Reasons for Action, the Aim of Belief, and the Aim of Action", Epistemic Norms, Oxford University Press, Oxford, 2014.

McHugh, C. y Whiting, D. "Recent Work on the Normativity of Belief», Analysis Reviews, 2014, Oxford Journals (on-line version).

${ }^{8}$ Unwin, N. Aiming at Truth, Palgrave MacMillan, London, 2007. 
mayoría tienden a considerar una relación constitutiva entre la creencia y verdad en términos de aspiración. También tomo en cuenta las ideas de Haack ${ }^{9}$ Hookway ${ }^{10}$, Kitcher $^{11} \mathrm{y}_{\text {Misak }}{ }^{12}$. David Papineau defiende una posición teleológica en la relación entre creencia y verdad. Para este autor no hay ninguna norma sobre la creencia aspirando a la verdad y las evaluaciones epistémicas no son siempre valiosas. Papineau no acepta "que siempre debamos tomar las evaluaciones epistémicas como valiosas comprometiéndonos con prescripciones sustanciales sobre lo que debe ser hecho»" ${ }^{13}$. Es más, la verdad ni siquiera es siempre valiosa desde perspectivas no epistémicas como las personales, morales o estéticas:

No siempre tiene valor personal, moral o estético evitar la creencia falsa. Y dado que rechazo cualquier clase distintiva de normatividad doxástica, acepto que hay casos en donde no hay nada malo en creer la falsedad ${ }^{14}$.

Parece que Papineau centra el problema no tanto en la sentencia la creencia aspira a la verdad o en la naturaleza de la creencia, sino más bien en la naturaleza de la verdad, no siendo siempre algo valioso. Papineau define la creencia en términos de diseño biológico, y más específicamente, la creencia ayuda a alcanzar y satisfacer deseos, generalmente, aunque no siempre, reflejando el mundo tal cual es:

Los deseos se relacionan con los resultados. Cada tipo de deseo tiene la función de generar acciones que conduzcan a un resultado específico, tal y como comida, o buen vino, o ver jugar a fútbol a los Tottenham Hotspurs. Por el contrario, las creencias no tienen resultados a establecer por sí mismas. Su función no es la de producir resultados específicos, sino ayudar a quien quiera que desee a seleccionar aquellas acciones que conduzcan a su satisfacción ${ }^{15}$.

Papineau expone claramente sus ideas al final de este artículo:

La evolución nos ha inculcado el hábito de ajustar nuestras creencias con la evidencia [...]. Pero esto no significa que debamos ajustar nuestras creencias. Si nada de valor

9 HaAck, S. «Concern for Truth: what it Means, why it Matters», Annals of the New York Academy of Sciences, 775(1), 1996, pp. 57-63.

${ }_{10}$ Hookway, C. «Truth, Reality, and Convergence», The Cambridge Companion to Peirce, Cambridge University Press, Cambridge, 2004, pp. 127-149.

${ }_{11}$ Kitcher, P. «On the Explanatory Role of Correspondence Truth», Philosophy and Phenomenological Research, 64(2), 2002, pp. 346-364.

12 Misak, C. «Deflating Truth: Pragmatism vs. Minimalism», The Monist, 1998, pp. 407425. Misak, C. Truth and the End of Inquiry: A Peircean Account of Truth, Oxford Philosophical Monographs, Oxford, 1988.

\footnotetext{
13 Papineau. Op. cit., p. 69.

${ }_{14}$ Ibidem, p. 68

15 Ibidem, p. 73.
} 
moral o personal o estético se perdiera, entonces no habría nada malo en ignorar la evidencia, incluso si no podemos ${ }^{16}$.

Algunos normativistas responderían que la norma doxástica de la creencia no se aplica a los resultados finales (la creencia refleja la verdad frente a la creencia falla a la hora de reflejar la realidad), sino en la naturaleza del proceso de la aspiración: no importa si nuestras creencias finalmente reflejan o no la realidad, sino que la voluntad del agente cuando cree es reflejar la verdad, siendo esta la norma ${ }^{17}$. Otros normativistas dirían que «obtener la verdad» es una consideración pragmática que lleva al agente a una acción satisfactoria. Por ejemplo, David Owens explica que «la función de la creencia es regular nuestras vidas emocionales» ${ }^{18}$. Sin embargo, lo hace desde una perspectiva doxástica: la creencia apunta a la verdad y como resultado obtenemos una acción satisfactoria. Es más, si la verdad y la acción satisfactoria están confrontadas, la normatividad doxástica prevalece (la creencia debe aspirar a la verdad). En su análisis de las concepciones pragmáticas de la creencia, Owens establece que «la función de la creencia es ser verdad y el valor de la creencia verdadera reside en motivar la acción exitosa, la acción que alcanza sus objetivos» ${ }^{19}$.

De una manera similar, Daniel Whiting ${ }^{20}$ defiende una opción normativa de la creencia aspirando a la verdad de una manera falsacionista: la creencia puede aspirar a la verdad y la creencia no debe aspirar a la falsedad. Whiting considera que la verdadera naturaleza normativa de la creencia es constitutiva y no evaluativa. En una popular analogía con el ajedrez, se tienen unos movimientos permitidos para cada pieza, así que podemos mover las piezas siguiendo estas reglas pero no debemos hacer lo contrario ${ }^{21}$. Claro está, esto no es lo único que se necesita para ganar al ajedrez: debemos hacer buenos movimientos. Pero esto último es una cuestión evaluativa que no define el juego del ajedrez. De una manera más general, la verdad es una característica constitutiva de la creencia (o más concretamente, de la aspiración de la creencia) y los resultados prácticos no definen la creencia sino que la evalúan.

Desde esta propuesta falsacionista, Whiting sugiere que el agente aspira a creer la verdad como consecuencia de algún tipo de aceptación previa de la verdad como norma de la creencia. Admite que algunas veces la norma de la verdad podría no ser suficiente para explicar las creencias finales, sino que otras consideraciones no epistémicas o no doxásticas se necesitarían: «Las consideraciones prácticas difícilmente pueden ser irrelevantes sobre si la creencia de uno puede figurar o no en

16 Ibidem, p. 79.

${ }^{17}$ Nótense las últimas palabras de Papineu en la cita anterior: «Incluso si no podemos [ignorar la evidencia]».

${ }_{18}$ Owens. Op. cit., p. 37.

19 Ibidem, p. 42.

20 Whiting. Op. cit., 2010, 2013a, 2013b, 2014.

${ }^{21}$ Whiting. Op. cit., 2010, 2013a. 
su razonamiento práctico sobre qué hacer» ${ }^{22}$. Así, la creencia no debe apuntar a la falsedad y las consideraciones prácticas son especialmente importantes para actuar.

Recientemente, Whiting ha profundizado en la dicotomía entre verdad y consideraciones prácticas a la hora de formar creencias: "Que los sujetos no toman las consideraciones prácticas para justificar las creencias parece desconcertante; después de todo, muchas de estas consideraciones tienden a manifestarse en asuntos más apremiantes que la verdad o la falsedad $^{23}$. «Dado que tenemos objetivos prácticos así como epistémicos, ¿por qué los últimos tienen que dominar cuando se trata de creer? $\aleph^{24}$. De manera más general, Whiting defiende las creencias correctas (desde el punto de vista doxástico), causadas por las evidencias y promoviendo o permitiendo condiciones para las acciones prácticas. También establece que centrarse en las acciones y razones prácticas explica mejor el compromiso de los agentes con las evidencias que centrarse solo en la verdad ${ }^{25}$, en tanto en cuanto «solo la evidencia puede tratarse como razón para creer ${ }^{26}$. Así, considera que la verdad y las cuestiones prácticas no están enfrentadas, sino relacionadas. Algunas veces, arguye que «el objetivo de creer solo lo que es una razón práctica genera el objetivo de creer solo lo que es verdad $\aleph^{27}$, esto es, creer en función de razones prácticas implica creer aspirando a la verdad, ya que lo que es verdad es práctico.

En otros ejemplos, las creencias apuntan a la verdad y no a las cuestiones prácticas. En el caso de que creer algo falso reportara beneficio al creyente -hoy es domingo pero creer que hoy no es domingo se le premiaría al creyente con $1000 €-$, entonces si las razones prácticas fueran las únicas para desarrollar una creencia el agente podría desarrollar esta creencia falsa, pero no puede. De hecho, puede decirse que lo práctico no es verdad en este caso y que la creencia aspira directamente a la verdad, contradiciendo que la aspiración a creer las razones prácticas para actuar genera la aspiración para creer la verdad. Aunque puede replicarse que la verdad es práctica incluso en estos $\operatorname{casos}^{28}$ (por ejemplo, creer la verdad, esto es, que es domingo, hace que el creyente no vaya al lugar de trabajo inútilmente), el resultado más práctico es creer que hoy no es domingo aunque lo sea. McHugh y Whiting consideran que en los casos de «wishful thinking estás influenciado por lo que te haría feliz creer, pero no puedes tratar esto como una razón para creer en tu deliberación ${ }^{29}$ : su única razón es la evidencia. Whiting finalmente apunta que

las consideraciones proporcionan razones para creer porque los sujetos aspiran a creer solo la verdad porque los sujetos aspiran a creer lo que son razones prácticas

22 Whiting. Op. cit., 2013 a, p. 194.

23 Whiting. Op. cit., 2014, p. 220.

${ }^{24}$ Ibidem, p. 223.

25 Ibidem, pp. 223-226.

${ }^{26}$ McHugh y Whiting. Op. cit., p. 5.

${ }^{27}$ Whiting. Op. cit., 2014, p. 226.

28 Whiting. Op. cit., 2014.

${ }^{29}$ McHugh y Whiting. Op. cit., p. 6. 
porque los sujetos aspiran a actuar y decidir siendo guiados solo por razones prácticas. La perspectiva epistémica no está en tensión con la perspectiva práctica, sino que está dictada y contenida en ella ${ }^{30}$.

Algo que interpreto de manera muy similar a la idea de Owens ${ }^{31}$ sobre la creencia, la verdad y el pragmatismo: conocer la verdad motiva acciones satisfactorias y efectivas. Más específicamente, la creencia aspira a la verdad (para Whiting, la creencia puede aspirar a la verdad y la creencia no debe aspirar a la falsedad) y la verdad normalmente genera resultados prácticos, así que la creencia aspira a los resultados prácticos. En palabras de Owens, para el pragmatista «decidimos qué pensar formando creencias debido básicamente a la misma razón por la que nos fijamos qué hacer formando una intención para hacerlo: actuar efectivamente» ${ }^{32}$. «Estamos autorizados a creer que $p$ si y solo si estamos autorizados a actuar como si $p$ fuera verdad (o tomar $p$ como razón para la acción) $\aleph^{33}$. Desde esta perspectiva, la fuente de cualquier posición normativista es la capacidad de producir estados y acciones útiles y prácticos. Sin embargo, Owens abre la puerta a la posibilidad de la verdad sin que esta implique acción efectiva o un estado útil, como en el ejemplo de una persona enferma terminal cuyo hijo es un terrorista odiado (lo más útil para esta persona sería creer lo falso, que su hijo es inocente). Esto significaría que esta posición pragmática sobre la creencia no siempre podría apoyar simultáneamente tanto el objetivo de la verdad como el objetivo de razones prácticas para la acción. Owens explora una definición más general de Pragmatismo por defecto: «Estar autorizado para creer que $p$ es estar autorizado para usar $p$ como asunción por defecto en el razonamiento práctico de uno $»^{34}$.

Sin embargo, con esta última posición surgen otros problemas: las creencias son transparentes, involuntarias e independientes del contexto, mientras que las asunciones no. La creencia es transparente, así que el creyente solo está motivado en su creencia por consideraciones relevantes para la verdad de la creencia: no podemos creer lo falso conscientemente y deliberadamente, si el agente se pregunta si creer que $p$ automáticamente se pregunta si $p$ es verdad. Precisamente los teleologistas explican este hecho apelando al objetivo de la verdad, mientras que los normativistas usan la norma de la verdad. Aceptaciones, aserciones y asunciones pueden incorporar otras consideraciones no doxásticas, como las pragmáticas. La creencia se relaciona automáticamente con la verdad (si creemos que $p$, entonces directamente y automáticamente tomamos $p$ como verdadero), mientras que las aceptaciones, las aserciones y las asunciones no. En otras palabras, el agente puede deliberadamente aceptar, asertar y asumir algo falso mientras que no puede creer algo falso deliberadamente. Un ejemplo popular es el del abogado: un abogado puede creer que su cliente es

\footnotetext{
30 Whiting. Op. cit., 2014, p. 235.

31 Owens. Op. cit., p. 15.

32 Ibidem, p. 43.

33 Ibidem, p. 42.

34 Ibidem, p. 45.
} 
culpable (involuntariamente e independientemente del contexto), pero podrá aceptar, asumir y asertar que es inocente durante el juicio ${ }^{35}$. Whiting también apunta a esta diferencia entre creencias y aserciones: mientras el agente cree automáticamente, la aserción implica reflexión. Esta diferencia es importante porque permite a muchos autores diferenciar entre la creencia aspirando a la verdad y la aserción aspirando a consideraciones pragmáticas. También significa que nuestras acciones no solo están motivadas por nuestras creencias sino que también pueden estarlo por nuestras aserciones y aceptaciones de una manera pragmática, abriendo una nueva puerta al pragmatismo: «La tesis de que debemos remplazar nuestras creencias, tal y como se entienden tradicionalmente, por lo que llamamos aceptaciones» ${ }^{36}$, porque «lo que es relevante es la adopción deliberada de un objetivo" ${ }^{37}$.

Por estas razonas Owens duda sobre el pragmatismo por defecto sobre las creencias y apoya su definición inicial: «En general las cosas irán mejor si basamos nuestros planes en el conocimiento antes que en la ignorancia ${ }^{38}$. Enfrentándose a casos como el de la persona terminal cuyo hijo es un odiado terrorista - «puesto que sentirse devastado no tiene ventajas sobre sentirse en paz, ¿cómo puede el conocimiento derivar su valor del hecho que nos requiere esto? ${ }^{39}-$, Owens hace referencia a la naturaleza normativa del conocimiento que él defiende: «Explicando dónde reside el valor del conocimiento, hemos establecido la autoridad de la norma del Conocimiento y así de las normas epistémicas que tenemos que satisfacer para alcanzar conocimiento» ${ }^{40}$. Estas normas epistémicas funcionan como convenciones.

De manera similar Kitcher ${ }^{41}$, Hookway ${ }^{42}$ y Misak ${ }^{43}$ aportan algunas ideas interesantes desde su análisis de Peirce: la realidad es externa e independiente de la mente, mientras que la verdad es una reflexión abstracta cuyo límite ideal es la realidad. Podemos decir que desarrollamos la verdad según consideraciones pragmáticas. Podría haber realidad sin verdad y podría haber verdad -incluso verdad social compartida- sin realidad. Ejemplos del primer caso serían descubrimientos que todavía no se han hecho. Ejemplos del último caso serían verdades sociales y asentadas que acabaron siendo falsas, como la de que la Tierra sea el centro del Universo. Nunca sabemos cuándo la investigación y la creencia se han desarrollado lo suficiente: una creencia asentada tomada como verdad y no la realidad es lo máximo que la investigación puede dar. Por otro lado, las creencias tienen que establecerse en términos de evidencias y los juicios sociales, y no debido a su consistencia interna, coherencia o voluntades personales y sociales. Todo esto implica una posición falsacionista

35 Unwin. Op. cit., p. 109.

${ }^{36}$ Ibidem, p. 163.

37 Ibidem, p. 175.

38 Owens. Op. cit., p. 40.

39 Ibidem, p. 51.

40 Ibidem, p. 52.

${ }^{41}$ Kitcher. Op. cit., 50.

42 Ibidem.

43 Misak. Op. cit., 1988, 1989. 
sobre las creencias y la verdad basada en la investigación rigurosa, y finalmente en la evidencia, la percepción, la experiencia y el compromiso público, prestando especial atención a los métodos científicos: «Lo que el pragmatista defiende es que cuando decimos que aspiramos a la verdad, lo que decimos es que, si una creencia satisface todos nuestros objetivos de investigación, entonces esta creencia sería verdadera ${ }^{44}$. Para Misak, la visión de Peirce de la creencia y la verdad implica algún tipo de normatividad. Pero esta normatividad no se relaciona con las creencias que se corresponden con la realidad, sino solo con un compromiso con la investigación correcta basada en la evidencia, la experiencia, la percepción y el juicio comunitario ${ }^{45}$ :

Los principios normativos no están siendo disimuladamente encubiertos aquí; están siendo reconocidos explícitamente como parte de lo que consideramos que es la creencia y la investigación. La normatividad que se deja todavía fuera es del siguiente tipo: las creencias verdaderas son creencias que todavía tienen la virtud de corresponder a la realidad, con "principios esenciales» o con "caracteres reales»"

La visión de Peirce de nuestro problema supone un intento de reconciliar realismo y pragmatismo: las creencias (basadas en la investigación) apuntan a la verdad en términos de éxito y uso, desarrollando creencias asentadas. Estas creencias asentadas apuntan a la realidad. Así este pragmatismo implica realismo, en tanto en cuanto admite una realidad externa como último paso del "proceso de aspiración", aunque nunca se culmine. Engel defiende el absolutismo doxástico y una visión normativa de la creencia contra el relativismo doxástico. Afirma que «el tipo correcto de razón para una creencia es la razón epistémica, y el tipo incorrecto de razón para la creencia es la pragmática» ${ }^{47}$. "Las creencias están gobernadas, normalmente, por un único tipo de razón, en concreto aquellas que son epistémicas -verdad y evidencia-» ${ }^{48}$. Esto significa que «el tipo correcto de razones son aquellas que están vinculadas con el objetivo o el contenido, mientras que el tipo incorrecto de razones son aquellas que están relacionadas con la actitud o el estado» ${ }^{49}$. De manera más clara:

Tomamos directamente la creencia como correcta porque es verdadera o basada en evidencias apropiadas, y no evaluamos su corrección con respecto a otros criterios, como la utilidad de la misma, su conformidad a la naturaleza, o el placer, o similares. En otras palabras, las razones epistémicas para la creencia parecen ser

${ }^{44}$ Misak. Op. cit., 1998, p. 410.

${ }^{45}$ De manera parecida, Susan Haack establece que «la verdad está internamente relacionada con los conceptos de creencia, evidencia e investigación. Creer que $p$ es aceptar $p$ como verdadera». НАACK. Op. cit., $\Phi 3$.

46 Misak. Op. cit., 1998, p. 65.

47 Engel. Op. cit., 2013a, p. 23.

${ }^{48}$ Ibidem, p. 51.

49 Ibidem, p. 24. 
el único tipo de razones que uno considera, y tiene que considerar, cuando uno forma una creencia ${ }^{50}$.

Engel también relaciona las aproximaciones teleológicas con hipotéticos valores instrumentales y con una variedad de objetivos de la creencia, y como resultado identifica estas opciones teleológicas con un relativismo: «¿Cómo distinguirá [el teleologista] su visión de una visión relativista? Para él parece claro que no siempre aspiramos a la verdad con nuestras creencias, sino más bien, al comfort " ${ }^{51}$. Este autor explica casos de wishful thinking como el de la persona enferma terminal de la siguiente manera:

La decisión del agente de no investigar [...] no es una excepción al objetivo de la creencia o a la norma, dado que lo que la última requiere es que cuando uno establece la consideración de una creencia para su verdad, entonces la creencia que forma tiene que ser verdadera ${ }^{52}$.

Así pues, el agente puede formar una creencia o no, pero una vez que la forma, tiene que apuntar a la verdad. Los ejemplos de wishful thinking serían creencias, ya que aunque estén formadas en base a evidencias e investigaciones pobres o nulas, el agente apunta apunta a la verdad, esto es, toma su creencia como verdadera. Haack llama pseudocreencias a estos «estados de lealtad obstinada hacia una proposición que uno entreve que es falsa, y de apego emocional a una proposición que uno no ha considerado" ${ }^{53}$. Esto parece apuntar a una redefinición de la creencia que no puede aplicarse a muchos casos populares y filosóficos en los que usamos la palabra "creencia». Si no investigamos más profundamente o no lo hacemos honestamente, el estado no puede considerarse una creencia. Sin embargo, Engel ofrece una descripción más detallada y una solución a este problema:

¿Ahora qué ocurre con los casos problemáticos donde no deliberamos explíitamente
y conscientemente sobre si creer que $p$, como en los wishful thinking, self-deception,
y otro tipo de creencias irracionales? [...]. Incluso aunque esta gente obviamente
no razona de manera consciente con y desde sus creencias de acuerdo a normas
de evidencia, es menos claro que no tenga ningún entendimiento de lo que una
creencia adecuada debería ser [...]. Así que no está claro que la norma de la verdad
no regule tácitamente el pensamiento en estos casos ${ }^{54}$.

Incluso si el wishful thinker tiene una creencia falsa, cree que esta creencia falsa apunta a la verdad. El wishful thinker está sujeto a una norma constitutiva de la creencia, su creencia continúa aspirando a la verdad, aunque se base en evidencias

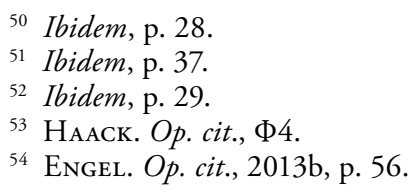


incorrectas. Unwin establece que la verdad, como aspiración de la creencia, podría ser valiosa hasta cierto punto, aunque «no está del todo claro cuánta importancia debe dársele a la búsqueda de la verdad ${ }^{55}$, como podemos ver en los casos de wishful thinking. De manera más clara:

Lo que parece que tenemos, más bien, es una situación en la que un deseo por la verdad tiene un rol central dentro de nuestra red de creencias-deseos, y es en primer lugar la presencia de este nodo particular en el sistema la que asegura que las consideraciones pragmáticas no nos llevan a caer en wishful thinking-al menos, no mucho $^{56}$.

La idea de Unwin podría expresarse de la siguiente manera: la creencia normalmente aspira a la verdad. Hay un deseo biológico práctico por la verdad, pero el valor de la verdad o la norma de la verdad no son lo suficientemente fuertes como para establecer que «la creencia siempre aspira a la verdad». Esto ofrece un análisis interesante de los casos de wishful thinking. Otro análisis de estas ideas podría ser que «la creencia siempre aspira a la verdad» y que «la creencia normalmente refleja la verdad». La diferencia aquí estaría en que hay un valor constitutivo o norma de la creencia apuntando a la verdad (la creencia siempre apunta a la verdad), pero no siempre satisface esta aspiración. Así, el wishful thinker aspiraría a la verdad, pero la verdad que considera no es la «real» (epistémica o socialmente aceptada): sencillamente falla a la hora de creer la «verdad real». Esta opción es la misma que la defendida por Engel ${ }^{57}$, y es la que también defiendo en este artículo.

\section{DAVIDSON, RORTY, CREENCIA Y VERDAD}

En esta sección examino las ideas de Davidson y Rorty sobre la verdad. Introduzco el concepto davidsoniano de creencia y analizo la verdad según Davidson, para posteriormente aplicar este análisis a los problemas de «la creencia aspirando a la verdad». Luego explico la visión de la verdad de Rorty y relaciono ambos autores. Finalmente muestro las conclusiones en términos del esquema teórico explicado en la anterior sección. En palabras de Davidson, «la creencia se construye para salvar la diferencia entre las sentencias tomadas como verdaderas por los individuos y las sentencias verdaderas (o falsas) para estándares públicos ${ }^{58}$. Es un concepto irreducible (no puede definirse en términos de otros conceptos) que el agente usa para desarrollar teorías consistentes y coherentes. Si no fuera así, sería un concepto

55 Unwin. Op. cit., p. 184.

56 Ibidem, p. 184.

57 Engel. Op. cit., 2013 b.

${ }^{58}$ Davidson, D. «Belief and the Basis of Meaning», Synthese, 27 (3-4), 1974, p. 321. 
prescindible: «¿Por qué ser tacaño para conceder premios?»" ${ }^{59}$. Esto nos da una idea de los pensamientos de Davidson sobre la creencia como concepto no prescindible que sirve como puente entre lo agencial y lo social.

Curiosamente, aunque son irreducibles, «los conceptos lingüísticos y semánticos son parte de una teoría intuitiva para organizar datos más primitivos, así que solo se puede obtener confusión si tratamos estos conceptos y sus supuestos objetos como si tuviesen vida propia por sí mismos» ${ }^{60}$. Conceptos como la creencia no son independientes (no son parte de una realidad externa) y están incrustados en una red, pero debemos continuar teorizando sobre ellos para encontrar las mejores teorías posibles. El punto cuando se tratan la creencia, la verdad y otros conceptos como el deseo, la aceptación, el conocimiento, la acción o la causa es relacionar todos ellos, y no intentar definirlos en términos de otros conceptos más claros, simples o fundamentales. Así, la verdad no puede entenderse simplemente como «asertabilidad garantizada»:

Las condiciones de asertabilidad garantizada se hacen tan duras que incluyen la verdad en sí misma, en cuyo caso la opción se vuelve circular, o la circularidad se evita haciendo las condiciones explícitas, y entonces se hace patente que una aserción completamente garantizada podría ser falsa ${ }^{61}$.

De manera contraria a los conceptos, la evidencia es externa y proporciona un punto para la interpretación. La base evidencial para la creación de conceptos consiste en hechos "sobre las circunstancias bajo las cuales los hablantes poseen sentencias" ${ }^{62}$, así que depende del contexto. Una vez que se tiene toda la evidencia posible, puede darse que permanezcan abiertas teorías alternativas sobre tal evidencia. La verdad no tiene aplicación o interés si no tiene una conexión empírica:

[Esta] cuestión empírica es cómo determinar, por observación e inducción, cuáles son las condiciones de verdad para los vehículos de la verdad empírica [...]. Por lo tanto veo el problema de conectar la verdad con el comportamiento observable humano como inseparable del problema de asignar contenidos a todas las actitudes ${ }^{63}$.

La verdad no es un objeto sino un concepto. Esta visión de la verdad nos permite establecer que diferentes creencias irreconciliables podrían ser consideradas como verdaderas simultáneamente. Esto es así porque no tenemos disponibles entidades apropiadas que puedan explicar por qué las sentencias verdaderas son verdaderas y las falsas son falsas: la verdad es irreducible. El concepto más cercano

59 Davidson, D. «Truth Rehabilitated», Truth, Language and History, Clarendon Press, Oxford, 2000, p. 17.

${ }^{60}$ Davidson, D. Op. cit., 1974, p. 311.

${ }^{61}$ Davidson, D. Op. cit., 2000, p. 8.

${ }^{62}$ Davidson, D. Op. cit., 1974, p. 320.

63 Davidson, D. «The Folly of Trying to Define Truth», The Journal of Philosophy, 93 (6), 1996, pp. 277-278. 
es el de evidencia, pero no es suficiente, en tanto en cuanto su tratamiento es social y subjetivo, y por tanto no tiene por qué tener una interpretación única. En palabras de Davidson, «lo mejor que podemos hacer es examinar, experimentar, comparar, y mantener la mente abierta. Pero no importa cuánto de profundo y de bien nosotros y las generaciones venideras lo hagan, nosotros y ellos tendremos creencias falibles» ${ }^{64}$. Los pragmatistas normalmente consideran que, en tanto en cuanto no podemos alcanzar la verdad, las creencias verdaderas son las mejor investigadas y las más exitosas. Davidson considera que la verdad como concepto irreducible y como aspiración es importante porque nos permite comprender sentencias y ser criaturas pensantes.

En resumen, para Davidson la creencia es irreducible, no puede definirse en términos de «asertabilidad garantizada», sus aplicaciones más útiles o cuestiones similares. Más aún, la verdad no es independiente de otras actitudes como los deseos. Aunque lo mejor que un agente puede hacer es investigar desde las evidencias para formar creencias que apunten a la verdad, estas creencias son siempre falibles. Es más, las creencias las construyen criaturas pensantes «para salvar la diferencia entre las sentencias tomadas como verdaderas por los individuos y las sentencias verdaderas (o falsas) para estándares públicos» ${ }^{65}$.

La interpretación que podemos hacer de «la creencia aspirando a la verdad» desde las ideas de Davidson es ambigua. Se puede considerar que la creencia aspira a la verdad de una manera normativa y, en tanto en cuanto estos conceptos son dependientes y relacionados (aunque irreducibles), se podrían explicar fenómenos como el wishful thinking considerando que los deseos entran también en juego. Sin embargo, en tanto en cuanto los agentes no pueden obtener la creencia infalible sino solo investigar desde las evidencias, y en tanto en cuanto las creencias ayudan a salvar el salto entre las verdades de los agentes y de las comunidades (algo que puede ser hecho de maneras muy diferentes), una aproximación teleológica basada en valores hipotéticos y flexibles, así como en esta "verdad deflactada» (la única que el agente puede obtener), parece apropiada. De manera parecida a Davidson, Rorty se centra especialmente en el análisis de la verdad. Aunque no puede decirse que Rorty afronte el problema en los términos expuestos (la verdad aspirando a la verdad), su tratamiento de la verdad es útil para este análisis y en algunos aspectos puede ponerse en las mismas líneas de pensamiento de Papineau y Unwin, tal y como muestro al final de esta sección.

Rorty, mientras analiza a Davidson, considera que su defensa de la irreducibilidad de conceptos como la verdad y la creencia está causada por un uso cautelar de estos términos ${ }^{66}$. Para Rorty, la verdad es reducible al uso y a la práctica, por lo que no hay mucho más que decir sobre la verdad en sí misma. De manera más amplia, Rorty rechaza el autoritarismo de conceptos como el bien, el destino, la

${ }^{64}$ Davidson, D. «The Structure and Content of Truth», The Journal of Philosophy, 87 (6), 1990 , p. 6.

${ }^{65}$ Davidson. Op. cit., 1974, p. 321.

${ }^{66}$ Rorty, R. «Response to Donald Davidson», Rorty and their Critics, Wiley-Blackwell Publishers, Hoboken, 2000b, p. 77. 
verdad y finalmente la realidad. También rechaza el objetivo de validez universal de la creencia y la verdad basada en un compromiso con la justificación a todos los agentes en cada comunidad en términos de «realidad».

Rorty también se opone a la idea de dos normas diferentes sobre la creencia: la de la «asertabilidad garantizada» y la de la verdad. Así, Rorty considera que «el hecho de que las creencias puedan ser justificadas sin ser verdad no supone que se esté recurriendo a dos normas» ${ }^{67}$, algo que sería útil para interpretar algunas creencias falsas, como los casos de wishful thiking. De hecho, esta diferencia entre normas es "un objetivo para los investigadores metafísicamente activos» ${ }^{68}$, mientras que Rorty se define a sí mismo (y a Davidson) como metafísicamente quietista ${ }^{69}$. Lo que Rorty propone es un concepto de verdad basado en la aptitud y la utilidad, que no necesitaría de ningún elemento extrarrealista o metafísico. El pragmatista podría defender que, aunque puede haber muchos objetivos en la creencia, ninguno de ellos es metafísicamente fundacional. Lo que el agente quiere mientras cree es justificar su creencia a la mayor audiencia posible. La diferencia entre la justificación como concepto pragmático y la verdad es que la primera no requiere de ninguna presuposición metafísica mientras que la segunda normalmente sí: «Una diferencia entre la verdad y la justificación es la que hay entre lo irreconocible y lo reconocible» ${ }^{70}$. No podemos pensar en principios y conceptos neutros y fundamentales, como la verdad entendida tradicionalmente. Esta diferencia puede estar causada por una sobreatención al estado de los hechos. El alcance y éxito de la justificación es solo temporal.

La relación entre verdad y justificación implica que la verdad normalmente presenta un uso cautelar. Esta diferencia nos permite tratar creencias falsas pero justificadas: "Utilizamos [la verdad] para recordarnos que la gente en distintas circunstancias - gente enfrentándose a distintas audiencias- podría no ser capaz de justificar la creencia que hemos justificado triunfantemente a todas las audiencias a las que nos hemos enfrentado [previamente], $\gg^{11}$. La habilidad para creer implica la habilidad para argumentar sobre qué creer. Para Rorty, todo lo que tenemos sobre las creencias y la verdad son audiencias competentes, aunque no podamos llegar a la comunidad entera. La objetividad no sería diferente de una intersubjetividad extendida. Una práctica social puede ser trascendida únicamente por otra práctica social, y no por conceptos fundamentales como la «verdad filosófica» o la «verdad real». Creencias y deseos están dentro de una red social: «Coherencia, verdad, y comunidad van juntos», pero esto no implica fundacionalismo o universalidad ${ }^{72}$. Comunidades diferentes pueden tener diferentes redes de creencias, deseos y verdades, siendo todas

${ }^{67}$ Ronty, R. Truth and Progress: Philosophical Papers (Vol. 3), Cambridge, Cambridge University Press, 1998, p. 27.

${ }_{68}$ Ibidem, p. 29.

69 Ibidem, pp. 29, 38.

70 Rorty, R. «Universality and Truth», Rorty and his Critics, Wiley-Blackwell Publishers, Hoboken, 2000a, p. 2.

${ }^{71}$ Ibidem, p. 4.

72 Ibidem, p. 16. 
ellas coherentes. La única cuestión en común para toda persona y comunidad que podemos entrever es precisamente la curiosidad, el deseo de investigar. Analizando las creencias falsas como los ejemplos de wishful thinking previamente expuestos, Rorty no considera que el agente esté bajo algún tipo de normatividad que le incite a aspirar a la verdad, sino que las creencias aspiran a la aptitud y la utilidad en sus contextos sociales y naturales:

¿Y qué sobre la afirmación de que todos los humanos desean la verdad? [...] la afirmación que todos ellos desean justificar sus creencias a algunos, aunque no necesariamente todos, otros seres humanos, y la afirmación que todos ellos quieren que sus creencias sean verdaderas. La primera afirmación es incuestionable, y la segunda dudosa ${ }^{73}$.

Así que Rorty explica la creencia en términos de uso, utilidad y justificación. Diferenciar entre las creencias que aspiran a la verdad y las creencias que aspiran simplemente a algo bueno para guiarnos es la posición de los realistas, y Rorty no encuentra motivo para ello. Al mismo tiempo, Rorty define la justificación en términos de utilidad dentro de un rango de posibilidades de uso. Los pragmatistas piensan que si algo no supone ninguna diferencia en la práctica, entonces no debería haber ninguna diferencia en filosofía. Esta convicción les hace sospechar de la diferencia entre justificación y verdad, dado que esta diferencia no supone ninguna diferencia en la acción. Si tengo dudas concretas y específicas sobre si una de mis creencias es verdadera, puedo resolver estas dudas preguntándome si está adecuadamente justificada ${ }^{74}$. Estos filósofos comparten una imagen de los seres humanos como máquinas construidas (por Dios o la Evolución) para, entre otras cosas, hacer las cosas correctas. Los pragmatistas queremos que nuestra cultura se libere de esta autoimagen y que se remplace por una imagen de máquinas que continuamente se ajustan a los comportamientos mutuos, y a su entorno, desarrollando nuevas formas de comportamiento. Estas máquinas no tienen ningún programa o función fijados ${ }^{75}$.

Podemos afirmar en términos de Rorty que la creencia aspira al uso y la utilidad (en sus contextos). En parte, esto coincide con el pensamiento de Papineau: «No tiene siempre valor personal, moral o estético evitar las creencias falsas [...] así pues, acepto que hay casos donde no hay nada erróneo en creer falsamente» ${ }^{76}$. Sin embargo, en sus citas, Papineau asume una verdad externa que juzga las creencias («creer falsamente»), algo que Rorty no hace. Nótese que esta visión es diferente a la que en ocasiones Owens y Whiting defienden: «Si es verdad, es bueno». Unwin también defiende un pragmatismo sobre la creencia y la verdad («no está claro cuánta importancia se le deba asignar a la búsqueda de la verdad $»^{77}$, pero al mismo

\footnotetext{
73 Ibidem, p. 4.

74 Rorty. Op. cit., 1998, p. 19.

75 Ibidem, p. 32.

76 Papineau. Op. cit., p. 68.

77 Unwin. Op. cit., p. 184.
} 
tiempo establece que un deseo común por la verdad epistémica aleja al agente del relativismo radical y previene al agente de caer continuamente en wishful thinkings.

\section{CONCLUSIÓN: DILEMAS Y PUNTOS CLAVE SOBRE LA PREGUNTA ¿POR QUÉ CREEMOS?}

He mostrado diferentes maneras de ajustar la verdad, la creencia y posturas pragmáticas. Actualmente, muchos autores analizan la cuestión anterior centrándose en la creencia y sus aspiraciones.

Owens considera que «la función de la creencia es ser verdadera y el valor de la creencia verdadera reside en motivar la agencia exitosa ${ }^{78}$. Lo que queremos cuando formamos creencias es actuar efectivamente. Owens también explora una postura pragmática más radical, en la que «estar autorizado para creer que $p$ es estar autorizado para usar $p$ como asunción por defecto en el razonamiento práctico de uno" ${ }^{79}$. Esta idea es muy similar a la de Rorty, en la que la creencia apunta al uso y la utilidad en sus contextos, pero Owens finalmente la matiza: «En general las cosas van mejor cuando basamos nuestros planes en el conocimiento mejor que en la ignorancia»" ${ }^{80}$. Esto supone un tipo de normatividad que Owens acepta: «La autoridad de la norma del Conocimiento" ${ }^{81}$. Engel, cuando trata de explicar los casos de wishful thinking, establece que el agente puede formar una creencia o no, pero una vez que la forma, esta debe basarse en la investigación correcta y la evidencia honesta. En tanto en cuanto los wishful thinking no se basan en la evidencia correcta y la investigación, no pueden ser tratados como creencias. Sin embargo, esta idea puede entenderse mejor si consideramos que el creyente siempre aspira a obtener la verdad, sin importar cuánto de buena es la evidencia y cuánto ha investigado. De manera similar, Whiting promueve una aproximación normativa a la creencia aspirando a la verdad: las creencias pueden aspirar a la verdad (de manera constitutiva). Pero, contrariamente a Engel, adopta una posición falsacionista: las creencias no deben aspirar a la falsedad. Cuando se forman las creencias, la norma es la de que no podemos desarrollar creencias aspirando a la mentira. Cuando se relacionan consideraciones pragmáticas y epistémicas, para Whiting creer la verdad implica creer por consideraciones prácticas: «Los sujetos aspiran a creer solo lo que es verdad porque los sujetos aspiran a creer solo lo que es una razón práctica [...]. La perspectiva epistémica no está en tensión con la perspectiva práctica sino dictada y contenida en ella ${ }^{82}$. Unwin advierte que no todas las creencias reflejan la verdad o el conocimiento y que «no está del todo claro cuánta importancia debe dársele a

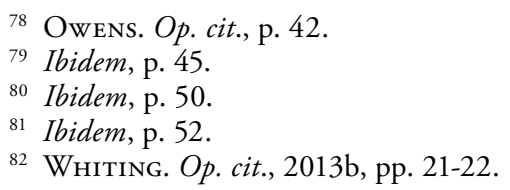


la búsqueda de la verdad $»^{83}$. Pero al mismo tiempo, este autor reconoce un papel especial de la verdad cuando formamos creencias: «Lo que parece que tenemos, más bien, es una situación en la que el deseo de la verdad tiene un rol central en nuestra red de creencias-deseos»" ${ }^{84}$. Hay un "valor frágil de la verdad», pero un valor. Esto puede interpretarse de dos maneras diferentes: «La creencia normalmente aspira a la verdad», aunque a veces no lo hace, ya que otros deseos son más fuertes que el deseo de verdad, o "la creencia siempre aspira a la verdad» y «la creencia normalmente refleja la verdad». Hay una gran diferencia entre estas dos posiciones: la última establece una conexión constitutiva entre creencia y verdad.

Para Davidson, la creencia sirve de puente entre las sentencias tomadas como verdad por los agentes y las sentencias tomadas como verdad desde estándares públicos. Considera que la verdad es un concepto irreducible, así que rechaza algunas de las principales definiciones pragmáticas de verdad en términos de uso y creencias exitosas. Por otro lado, la verdad de Davidson no es independiente: la verdad y la creencia están relacionadas con otras actitudes y estados como los deseos, formando una red. Lo mejor que un agente puede hacer es centrase en las evidencias para formar creencias, aunque sean siempre falibles. Rorty ofrece un análisis interesante de la verdad que puede ser aplicado a la sentencia de Williams «la creencia aspira a la verdad». La verdad se explica en términos de justificación, y finalmente, en términos de uso y utilidad en sus contextos. Así, podemos aplicar este análisis de la verdad a nuestro problema inicial: «La creencia aspira al uso y a la utilidad (en sus contextos)». En algunos aspectos, estas ideas son similares a las de Papineau y a las de Unwin, aunque la posición de Rorty es más radical: no admite ningún tipo de verdad epistémica ni de realidad externa.

Estas aproximaciones diferentes para ajustar la creencia, la verdad y consideraciones pragmáticas presentan algunos problemas y dilemas, y también ofrecen algunos puntos clave. A grandes rasgos, se pueden considerar dos posiciones principales: la creencia aspira a la verdad (considerada como realidad o conocimiento epistémico) y la creencia aspira a cuestiones prácticas ${ }^{85}$. Finalmente, algunos autores (Owens, Whiting, McHugh) intentan demostrar que las perspectivas epistémica y pragmática no están efrentadas, estableciendo que el objetivo epistémico implica el objetivo pragmático.

Si consideramos que la creencia aspira a la verdad, podemos encontrar algunos contraejemplos, como los de wishful thinking: puedo creer que le gusto a la chica que me gusta, aunque tenga evidencia opuesta; puedo creer que los Chicago Bulls ganarán la NBA, aunque la evidencia muestre que serán los Boston Celtics los que ganarán. La madre cuyo hijo es un terrorista odiado cree que no lo es, a pesar de la evidencia. En estos casos la creencia no refleja la verdad. Si aceptamos una relación

83 Unwin. Op. cit., p. 184.

${ }^{84}$ Ibidem, p. 184.

${ }^{85}$ Debe notarse que en función de los autores estas sentencias pueden significar cosas diferentes (algunas veces las mismas sentencias se aplican a aproximaciones normativas y teleológicas). 
constitutiva entre creencia y verdad, podemos decir que estas creencias fallan a la hora de alcanzar su objetivo: todas las creencias emergen bajo una norma de la verdad y algunas veces simplemente no pueden alcanzar su objetivo, aunque este objetivo está siempre ahí. Hay una norma constitutiva en la creencia aspirando a la verdad ${ }^{86}$, algo que se sigue muy bien desde las posturas realistas de defensa de un contenido metafísico en la relación entre creencia y verdad, siguiendo la crítica de Rorty. Por otro lado, para teleologistas como Papineau esta norma constitutiva puede analizarse también desde una posición biologicista, deflactando su normatividad. Podemos argumentar también que los casos de wishful thinking son creencias que aspiran a la verdad en base a evidencias falsas o pobres, pero igualmente evidencias: veo a la chica que me gusta tocándose el pelo cuando me ve; recuerdo las estadísticas de la NBA de 1961, que refuerzan la posibilidad de que sean los Chicago Bulls los que ganen; la madre recuerda que su hijo ayudó a un vagabundo cuando tenía cinco años. Esta posición apoyaría la existencia de una relación constitutiva entre creencia y verdad, e incluso la necesidad de evidencia para el desarrollo de creencias.

Si no aceptamos esta relación constitutiva, podemos decir que las creencias no tienen por qué aspirar a la verdad y en cambio podemos decir que simplemente aspiran a consideraciones pragmáticas (las creencias aspiran a su justificación). Así que cuando creo que la chica me quiere y que los Chicago Bulls van a ganar la NBA o cuando la madre cree que su hijo es inocente, lo hacemos para obtener un beneficio emocional y, en último término, porque es lo más práctico. Sin embargo, esta opción también presenta contraejemplos. Si fuera a ganar $1000 €$ por creer que hoy no es domingo y si la creencia aspirara a lo práctico, en tanto en cuanto ganar $1000 €$ es práctico, debería creer que hoy no es domingo. Pero la creencia es transparente y no podemos decidir nuestras creencias voluntaria y automáticamente. Podemos aceptar que hoy no es domingo para de esta manera obtener el premio. Pero la aceptación (voluntaria, dependiente del contexto y no transparente) no es una creencia. Como resultado de ello, bastantes autores han relacionado el pragmatismo con las aceptaciones: tenemos que convertir nuestras creencias en aceptaciones bien pensadas, sin negar que ambas son actitudes proposicionales diferentes. En este sentido, la transparencia y la independencia del contexto de las creencias son buenos argumentos para la defensa de una relación constitutiva entre creencia y verdad en términos de la aspiración de la creencia hacia la verdad.

De manera bastante sugerente, se puede ofrecer un argumento temporal para la opción de la creencia aspirando a la verdad: «La integridad intelectual es instrumentalmente valiosa, porque, a largo plazo y en términos generales, hace avanzar la investigación, y la investigación exitosa es instrumentalmente valiosa» ${ }^{87}$. "Incluso si la adecuación empírica condicionada pragmáticamente es suficiente para garantizar el funcionamiento en cualquier momento particular, algo más se necesita para ase-

86 Owens. Op. cit., p. 52.

87 Haаск. Op. cit., $\Phi 10$ (cursiva ańadida). 
gurar que la adaptación a largo plazo y la evolución pueden ocurrir efectivamente» ${ }^{88}$. Aunque a veces a corto plazo es más útil no creer la verdad y así actuamos, como en los casos de wishful thiking, a largo plazo es más práctico y mejor para nuestra adecuación emocional conocer la verdad: cuanto antes me dé cuenta de que la chica no me quiere, antes podré seguir con mi vida sin prestarle atención; cuanto antes me dé cuenta de que los Chicago Bulls no van a ganar la NBA, más leve será mi desilusión. Puede replicarse que en casos como el de la madre del terrorista odiado siempre es mejor para ella creer la mentira, en tanto en cuanto es lo más práctico. El normativista podría argumentar que en estos casos extrańos jugamos con la inexistencia o la limitación de la dimensión temporal, ya que el agente (la madre) fallecerá pronto. Este argumento temporal se ajusta bien a las opciones que defienden la reconciliación entre las perspectivas epistémica y pragmática que algunos autores promueven (Owens, Whiting, McHugh). En sus términos, lo que es verdad es práctico, así que la perspectiva epistémica está incluida en la pragmática. Si mostramos contraejemplos en los que lo práctico no es verdad (ganar $1000 €$ por creer que hoy no es domingo), estas posiciones podrán replicar que a largo plazo creer la verdad nos previene de muchos problemas (ir a trabajar cuando no debemos, cuidar a nuestros hijos en tanto en cuanto no están en la escuela...). Pero en mi opinión, una opción pragmática elegiría antes ganar los $1000 €$ en tanto en cuanto los resultados de creer la mentira son hipotéticos y menos prácticos.

También podemos centrarnos en una opción normativa sobre la creencia que no esté basada en la verdad sino en las cuestiones prácticas: la creencia debe aspirar a las cuestiones prácticas. Sin embargo, esta opción no resistiría algunos ejemplos como los previamente mencionados. Los autores más próximos a esta postura no consideran que la posible aspiración pragmática de la creencia esté bajo una norma, sino que la explican en términos de evolución y diseño biológico.

Otro problema (relacionado con aquel de las aceptaciones y la transparencia de la creencia) para la opción de la creencia aspirando a consideraciones pragmáticas es que en algunos contextos es útil adoptar algunas creencias mientras que en otros contextos lo útil es adoptar justo las creencias contrarias a las iniciales: un biólogo cristiano evolucionista podría creer en la evolución mientras está trabajando y creería en el génesis mientras está en la iglesia los domingos. Autores como Rorty, que rechazan las opciones normativas y el realismo, dirían que «todo lo que el pragmatista religioso necesita hacer es ser razonable, mantener su religión fuera de sus actividades científicas y políticas [...] la reconciliación sería solo necesaria si la creencia en ambas le llevara a algún tipo de incomodidad social ${ }^{89}$. Sin embargo, la creencia es transparente e independiente del contexto. Es importante notar que el hecho de que la creencia sea independiente del contexto no implica que no dependa del entorno social y de la comunidad. Esto es, las creencias pueden cambiar de comunidad a comunidad en sus

${ }^{88}$ Unwin. Op. cit., p. 147 (cursiva añadida).

${ }^{89}$ Rorty, R. "Something to Steer by" [Review of the book John Dewey and the High Tide of American Liberalism by A. Ryan], London Review of Books, 18 (12), 1996. 
contextos (por ejemplo, la comunidad de biólogos evolucionistas y la comunidad de católicos), pero las creencias no cambian para un mismo agente según sus distintos contextos a no ser que nuevos contextos aporten nuevas evidencias (por ejemplo, el abogado que cree que su cliente es culpable sin importar el contexto, aunque acepta su inocencia durante el juicio). Así, el hecho de que diferentes contextos personales puedan proporcionar nuevas evidencias más fuertes tampoco debería confundirse con la creencia siendo independiente del contexto.

En resumen, ambas posiciones aparentemente presentan contraejemplos. Por un lado, la opción de que «la creencia aspira a la verdad» se puede criticar con ejemplos de wishful thinking donde la creencia no refleja la verdad, el conocimiento o la realidad, y sin embargo puede ser útil. No obstante, se puede replicar que la naturaleza constitutiva de la creencia aspirando a la verdad se basa en una disposición motivacional interna que empuja al agente a reflejar el mundo tal cual es (puede que en ocasiones infectada por hechos conativos) pero incluso en estas creencias hay un objetivo de la verdad: esto es, el agente considera verdad aquello que cree. Por otro lado, la opción de que "la creencia aspira a lo práctico» se enfrenta al problema de que la creencia no puede desarrollarse automática y voluntariamente, así que no siempre podemos creer lo práctico: no podemos creer que hoy es domingo para ganar $1000 €$ si hoy no es domingo.

Una tercera opción para este debate sería relacionar las perspectivas pragmáticas y epistémicas, estableciendo que lo que es verdad es práctico (Owens, Whiting, McHugh): «La creencia aspira a la verdad» y, en tanto en cuanto la creencia es práctica, «la creencia aspira a consideraciones prácticas». Pero lo verdadero no siempre es práctico y lo práctico no siempre es verdadero: es práctico para mí creer que hoy no es domingo para poder ganar $1000 €$. Se podría decir que generalmente y a largo plazo lo más útil es conocer y creer la verdad: hoy es domingo, y así no tengo que ir al trabajo y tengo que cuidar a mis hijos al no estar en la escuela. Pero sobre esta creencia, la fuerza pragmática de ganar $1000 €$ es mayor que los hipotéticos problemas derivados de tener la creencia falsa y de que no sea domingo. No podemos decir que la verdad es práctica en estos casos, o al menos que sea lo más práctico.

Una manera mucho más adecuada para incorporar el pragmatismo al análisis de la creencia y sus aspiraciones es considerar el remplazo de las creencias por aceptaciones, cambiándolas dependiendo de los contextos para obtener las soluciones más útiles y prácticas. Pero las aceptaciones no son propiamente creencias, en tanto en cuanto no son transparentes, ni automáticas, ni involuntarias ni independientes del contexto. 